\title{
Sulforaphane Activates Brain-Derived Neurotrophic Factor (BDNF) Transcription by NF-E2-Related factor-2 (Nrf2), Showing Prevention Effects on the Microglial of Stressed Mice
}

\author{
Rui Tang \\ Jinan University \\ Qianqian Cao \\ Jinan University \\ Lujuan $\mathrm{He}$ \\ Jinan University \\ Pengfei Du
}

The Second Hospital of Jiaxing: The Second Affiliated Hospital of Jiaxing University

\section{Gang Chen}

Jinan University

\section{Rao Fu}

Sun Yat-Sen University

\section{Fei Xiao}

Jinan University

Yirong Sun

Chinese Academy of Sciences

Ji-chun Zhang ( $\nabla$ jczhang@jnu.edu.cn )

Jinan University

\section{Research}

Keywords: SFN, Nrf2, BDNF, transcription

Posted Date: November 4th, 2020

DOl: https://doi.org/10.21203/rs.3.rs-100350/v1

License: (c) (i) This work is licensed under a Creative Commons Attribution 4.0 International License.

Read Full License 


\section{Abstract}

Background: Accumulating evidence suggested that sulforaphane (SFN) showed prevention effects in stressed mice by activating the NF-E2-related factor-2 (Nrf2). However, the mechanism remains poorly understood. The present study aimed to investigate whether the prevention effects of SFN in stressed mice by activating brain-derived neurotrophic factor (BDNF) transcription on the microglial and revising abnormal dendritic spine morphology.

Methods: In vitro study, we performed the Luciferase assay, Chromatin immunoprecipitation assay, Immunofluorescence, and Western blot to investigate whether SFN could activate Nrf2, resulting in BDNF transcription on the BV2 cell. In vivo study, we employed the Social defeat stress mice model, Behavior test, Western blots, Immunofluorescence, and Enzyme-linked immunosorbent assay to further explore whether the prevention effects of SFN in stressed mice by activating BDNF transcription on the microglial and revising abnormal dendritic spine morphology.

Results: First, we found that SFN could activate Nrf2, resulting in Nrf2 binding to the BDNF exon I promoter, leading to BDNF protein expression on the BV2 cell. Second, SFN attenuated the decreased levels of Nrf2, and increased levels of MeCP2 expression, revising abnormal BDNF expression in the stressed mice. Third, we further found that the SFN could attenuate the decreased levels of Nrf2 and increased levels of MeCP2 expression on the microglial and revising microglial dysfunction in the stressed mice. Fourth, SFN could attenuate the increased levels of pro-inflammatory cytokine and the decreased levels of anti-inflammatory cytokine release in the stressed mice. Finally, SFN showed prevention effects and revised abnormal dendritic spine morphology in the stressed mice.

Conclusions: Therefore, the prophylactic effects of SFN in the stressed mice by activating BDNF transcription on the microglial and revising abnormal dendritic spine morphology.

\section{Background}

Sulforaphane (SFN: 1-isothiocyanato-4-methylsulfinylbutane) is an organosulfur compound, which is found in cruciferous vegetables, such as broccoli Brussels sprouts, and cabbage. Recently studies found SFN showed antioxidant and anti-inflammatory effects [1,2]. The mechanism for the antioxidant and anti-inflammatory effects of SFN is thought to be mediated by activating the NF-E2-related factor-2 (Nrf2) results in Phase II detoxification enzymes and antioxidant proteins transcription [3-7]. Our previous study found that intraperitoneally (IP) injection of SFN showed protection effects in lipopolysaccharide (LPS) and chronic social defeat stress (CSDS) models of depression by activating Nrf2 signaling and brainderived neurotrophic factor (BDNF) and tropomyosin-receptor-kinase B (TrkB) signaling $[2,8]$. Moreover, Dietary intake of glucoraphanin (a precursor for SFN) also showed prevention effects in the CSDS mice model by activating BDNF/TrkB signaling [8]. Taken together, these results suggested that SFN may have the function of prevention effects in depression animal models by activating Nrf2 and BDNF/TrkB signaling. 
A gradually increasing number of studies supported that the signaling of BDNF and TrkB are involved in the pathogenesis of depression and the therapeutic mechanisms of antidepressants [9-17]. Decreasing BDNF levels in the medial prefrontal cortex (MPFC) and hippocampus cause depression-like phenotypes [18-21], whereas the enhanced BDNF expression in the MPFC and hippocampus contribute to the resilience to inescapable electric stress and social defeat stress [22, 23]. Nrf2 is a key transcription factor that regulates antioxidant and anti-inflammatory responses. Recently study found that Nrf2 also plays a role in the pathogenesis of depression by affecting BDNF/TrkB signaling $[2,8,24]$. Therefore, it is very interesting to detail study the crosstalk of Nrf2 and BDNF/TrkB signaling in depression. Because Nrf2 is a key transcription factor for Phase II detoxification enzymes and antioxidant proteins. Therefore, we hypothesized that Nrf2 may function as a transcriptional activator for BDNF. The depression-like phenotypes caused by chronic stress associated with suppressed BDNF transcription, which enrolled the decreased Nrf2 and enhanced methyl-CpG binding protein-2 (MeCP2) (BDNF transcriptional repressor) [25] expression on the microglial. The prevention effects of SFN in stressed mice by activating Nrf2, resulting in BDNF transcription on the microglial.

In the current study. First, we found that SFN could activate Nrf2, resulting in Nrf2 binding to the BDNF exon I promoter, leading to BDNF protein expression on the BV2 cell (microglial). Second, SFN attenuated the decreased Nrf2 and increased MeCP2 protein expression, revising abnormal BDNF protein expression in the stressed mice. Third, we further found that the SFN could attenuate the decreased levels of Nrf2 and increased levels of MeCP2 expression on the microglial and revising microglial dysfunction in stressed mice. Fourth, SFN could attenuate the increased levels of pro-inflammatory cytokine and the decreased levels of anti-inflammatory cytokine release in stressed mice. Finally, SFN showed prevention effects in stressed mice and revised abnormal dendritic spine morphology. Therefore, the prophylactic effects of SFN in stressed mice by activating BDNF transcription on the microglial and revising abnormal dendritic spine morphology.

\section{Methods}

\section{Mice, cell lines, antibody information, and drug treatment}

Male adult C57BL/ 6 mice (8 weeks old, 20-25 g each, Guangdong Experimental Animal Center), CD1 mice (14 weeks old, 40-45 g each, Guangdong Experimental Animal Center), and male adult Thy1-Yellow fluorescent protein (YFP) mice were used in experiments. The animals were housed under controlled temperature and kept on a 12-h light/dark cycle (lights on between 07:00 and 19:00), with ad libitum access to food and water. The protocol was approved by the Jinan University Institutional Animal Care and Use Committee. All experiments were carried out following the Guide for Animal Experimentation of Jinan University. HEK293 or BV2 cells were cultured in high-glucose Dulbecco's Modified Eagle Medium (DMEM) supplemented with 10\% fetal bovine serum (Excell Bio.) and penicillin (100 units $/ \mathrm{mL}$ )streptomycin $\left(100 \mu \mathrm{g} / \mathrm{mL}\right.$ ) (all from Hyclone). Cells were incubated at $37^{\circ} \mathrm{C}$ in a humidified incubator containing $5 \% \mathrm{CO}_{2}$. The following antibodies were used for experiments Nrf2 (ab137550), MeCP2 
(ab2828), BDNF (ab108319), CD11b (Invitrogen 13-0112-82), iNOS (Invitrogen PA3-030A) and CD206 (MYBIOSOURCE MBS215669). The beta-actin antibody was purchased from EarthOx.

Lipopolysaccharide (LPS, 1 mg/ml for BV2 cell; L-4130, serotype 0111:B4, Sigma-Aldrich). SFN (10 mg/kg; MedChemExpress, Shanghai, China) was dissolved in distilled water containing $10 \%$ corn oil. SFN $(10 \mathrm{mg} / \mathrm{kg}$ ) was administered intraperitoneally (i.p.) to mice before the social defeat stress $30 \mathrm{~min}$ for 10 days. The dose of LPS and SFN was selected as previously reported $[8,26]$.

\section{Chronic social defeat stress (CSDS)}

For the CSDS depression model, the C57BL/ 6 mice were defeated by differently CD1 mice for 10 min total of 10 days. After the social defeat session, the CD1 mouse and C57BL/6 mice or Thy 1-YFP mice were housed in the half cage that separated by using a perforated Plexiglas divider, which can allow visual, olfactory, and auditory contact in the 24 hours. The C57BL/ 6 mice or Thy1-YFP mice have raised separately after finish the last session of social defeat. The social interaction test was performed to test the mice that were susceptible and unsusceptible.

For the social interaction test, an open box $(42 \times 42 \mathrm{~cm})$ was used, which has an interaction zone including a mesh-plastic target box $(10 \times 4.5 \mathrm{~cm})$ and two opposing corner zones. The two parts were used for this test (no social target and social target). For the no social target, the test mouse was placed into an open field arena for 2.5 min with no social target (no CD1 mouse) in the mesh-plastic target box. After no social target test, the mouse was placed into the open field arena again in the second 2.5 min with a social target (a novel CD1 mouse) in the mesh-plastic target box. The residence time in the interaction zone was counted by using the stopwatch, the time of ratio for social target, and no social target was calculated. About $70 \%$ of mice were susceptible after social defeat stress.

\section{Sucrose preference test}

The mice were habituated to a $1 \%$ sucrose solution for $48 \mathrm{~h}$ before the test day. And then the mice were deprived of water and food for $4 \mathrm{~h}$, followed by a preference test with water and $1 \%$ sucrose for $1 \mathrm{~h}$. The bottles containing water and sucrose were weighed before and at the end of this period and the sucrose preference (\%) was determined.

\section{Luciferase assay}

HEK293 cells were transfected with BDNF exon I, II and IV luciferase reporter together in 6-wells plates, pRL-TK Renilla luciferase plasmid (Promega), and different kinds of drugs, plasmids, or siRNA., the cells were collected and subjected to the dual-luciferase reporter assay kit (Promega) according to the manual after following transfection for $24 \mathrm{~h}$.

\section{Chromatin immunoprecipitation (ChIP) assay}


The cells were subjected to the ChIP assay protocol in the manual of the SimpleChIP® Enzymatic Chromatin IP Kit (Cell signaling), after transfection or treatment with certain plasmids or drugs. In the Chip assay, $7.5 \mu \mathrm{g}$ of Nrf2 antibody (Abcam) was added to the homogenate for the sample. In the PCR analysis, the BDNF exon I specific primers were used for the amplification of the promoter region. The primer sequences were: forward 5' GGCTTCTGTGTGCGTGAATTTGC 3'; reverse 5' AAAGTGGGTGGGAGTCCACGAG'. The PCR sample was resolved on a $2 \%$ agarose gel and sequenced after 35 cycles of PCR (denature at $95^{\circ} \mathrm{C}$ for $30 \mathrm{~s}$, anneal at $58^{\circ} \mathrm{C}$ for $30 \mathrm{~s}$, and extend for $30 \mathrm{~s}$ at $72^{\circ} \mathrm{C}$ ).

\section{Immunofluorescence staining}

The mice were anesthetized with sodium pentobarbital and perfused transcardially with $10 \mathrm{ml}$ of isotonic saline, followed by $40 \mathrm{ml}$ of ice-cold $4 \%$ paraformaldehyde in $0.1-\mathrm{M}$ phosphate buffer $(\mathrm{pH} 7.4)$. The brain samples were collected after perfused and postfixed overnight at $4^{\circ} \mathrm{C}$. The $50-\mu \mathrm{m}$ thick serial coronal sections of brain tissue were cut in ice-cold, 0.01-M phosphate-buffered saline ( $\mathrm{pH} 7.5)$, using a vibrating blade microtome (VT1000S, Leica Microsystems AG, Wetzlar, Germany). For the staining, the cells or mice brain sections were incubated with $3 \%$ hydrogen peroxide at room temperature for 10 minutes after the following fixing by $4 \%$ paraformaldehyde-fixed. And then the sections were blocked by a blocking solution for $1 \mathrm{~h}$ and incubated with primary antibodies overnight. The next day, the Alexa Fluor 488 or 568 conjugated isotype-specific secondary antibody was incubated for $1 \mathrm{~h}$ at room temperature. Images were then collected with an Olympus confocal microscope. The fluorescence intensity was quantified using Fiji/Image J.

\section{Enzyme-linked immunosorbent assay}

The blood samples were obtained via a cardiac puncture on 13 days after CSDS. The serum samples were obtained from blood by using centrifuging at $2000 \mathrm{~g}$ for $20 \mathrm{~min}$.

blood was centrifuged at $2000 \times \mathrm{g}$ for $20 \mathrm{~min}$ to generate serum samples. The serum samples were diluted 10-fold with ELISA (enzyme-linked immunosorbent assay) diluent solution. The serum levels of tumor necrosis factor- $a$ (TNF-a), interleukin-1 $\beta$ (IL-1 $\beta$ ), interleukin-6 (IL-6), interleukin-10 (IL-10) and interleukin-4 (IL-4) were measured using a Ready-SET-Go ELISA kit (eBioscience) according to the manufacturer's instructions.

\section{Western blotting assay}

The cell and mice brain sample was lysed in RIPA buffer $(20 \mathrm{mM} \mathrm{pH} 7.5$ Tris-HCl, $150 \mathrm{mM} \mathrm{NaCl}, 1 \mathrm{mM}$ $\mathrm{Na}_{2}$ EDTA, $1 \mathrm{mM}$ EGTA, 1\% Triton, $2.5 \mathrm{mM}$ sodium pyrophosphate, $1 \mathrm{mM}$ beta-glycerophosphate, $1 \mathrm{mM}$ $\mathrm{Na}_{3} \mathrm{VO}_{4}, 1 \mu \mathrm{g} / \mathrm{ml}$ leupeptin, $1 \mathrm{mM}$ phenylmethylsulfonyl fluoride) on ice for $30 \mathrm{~min}$. Cell lysates or brain lysates were then centrifuged at $13,000 \times \mathrm{g}$ for $30 \mathrm{~min}$ at $4{ }^{\circ} \mathrm{C}$. The supernatant was collected, and protein concentration was determined using a Coomassie Brilliant Blue protein assay kit (Bio-Rad). The same amount of the supernatant was boiled in SDS loading buffer. After SDS-PAGE, the samples were transferred to a polyvinylidenedifluoride (PVDF) membrane. The membranes were blocked in $2 \%$ BSA for 
$1 \mathrm{~h}$ at room temperature and then incubated with the primary antibody (The concentration is selected with the manufacturer's instructions) at $4{ }^{\circ} \mathrm{C}$ overnight. The next day, the blots were incubated with an antimouse (1:5000) or anti-rabbit (1:5000) secondary antibody. Images were captured with a Tanon-5200CE imaging system (Tanon, Shanghai, China), and immunoreactive bands were quantified.

\section{Dendritic spine analysis}

After the social interaction test, the Thy1-YFP mice were deeply anesthetized with sodium pentobarbital and perfused transcardially with $10 \mathrm{ml}$ of isotonic saline, followed by $40 \mathrm{ml}$ of ice-cold $4 \%$ paraformaldehyde in 0.1-M phosphate buffer ( $\mathrm{pH} 7.4)$. Brains were removed from the skulls and postfixed overnight at $4^{\circ} \mathrm{C}$. For dendritic spine analysis, $50-\mu \mathrm{m}$ thick serial coronal sections of brain tissue were cut in ice-cold, 0.01-M phosphate-buffered saline ( $\mathrm{pH} 7.5)$, using a vibrating blade microtome (VT1000S, Leica Microsystems AG, Wetzlar, Germany). The sections were mounted on gelatinized slides, dehydrated, cleared, and coverslipped under Permount ${ }^{\circledR}$ (Fisher Scientific, Fair Lawn, NJ, USA). Next, sections were imaged, and dendritic spine density was quantified in $10 \mu \mathrm{m}$ of each dendritic in a blinded manner.

\section{Statistical analysis}

The data are shown as the mean \pm standard error of the mean (S.E.M.). The data were analyzed using PASW Statistics 20 (formerly SPSS statistics; SPSS). All data were analyzed using a one-way analysis of variance (ANOVA), followed by the post hoc Fisher LSD test. $P$ values $<0.05$ were considered statistically significant.

\section{Results}

\section{SFN activates BDNF transcription, resulting in BDNF protein expression on the BV2 cell}

Previously study found that SFN could increase BDNF protein expression in mice brain $[2,8]$. In the current study, we aim to explore whether SFN could promote BDNF expression by affecting of $B d n f$ transcription on the microglial. First, we analyzed the DNA sequences of the promoter regions in mouse Bdnf exon I, II, and IV promoter by using luciferase assay. The luciferase assay found that SFN could activate the Bdnf exon I promoter. The activation of $B d n f$ exon I induced by SFN could be blocked by siRNA-Nrf2, suggesting SFN could activate Nrf2 results BDNF transcription (Fig. 1A). To further confirm whether SFN by activating Nrf2 results in BDNF transcription, we carried out a ChIP assay with BV2 cells treated with SFN by using an Nrf2-specific antibody or control IgG. PCR analysis with genomic DNA immunoprecipitated using the Nrf2 antibody demonstrated that SFN promoted Nrf2 interaction with the Bdnf exon I promoter more than the control IgG (Fig. 1B). Immunofluorescence staining revealed that LPS treatment caused the redistribution of $\mathrm{Nrf} 2$ and MeCP2 for the nucleus in BV2 cells. We found that more MeCP2 within the nucleus and the more diffused nuclear staining pattern of Nrf2 in vehicle-treated cells became more punctate upon LPS treatment, and this phenomenon could revise by SFN treatment 
(Fig. 1C). Western blot results showed that LPS significantly decreased Nrf2 and BDNF protein expression, and increased the Mecp2 protein expression. SFN significantly attenuated the reduction of Nrf2 and BDNF protein expression after LPS administration and increased levels of MeCP2 protein expression in the BV2 cell (Fig. 1D-G). These results suggest that Nrf2 is a transcriptional activator for BDNF, and SFN by activating Nrf2 promoted BDNF expression by binding with Bdnf exon I promoter on the BV2 cell.

\section{SFN attenuates the decreased levels of Nrf2 and increased levels of MeCP2 expression, revising abnormal BDNF expression in the stressed mice}

By using western blot, we found that social defeat stress significantly decreased the Nrf2 and BDNF protein expression, and increased the MeCP2 protein expression in the MPFC and hippocampus of stressed mice (Fig. 2A and B). SFN significantly attenuated the reduction of the Nrf2 and BDNF protein expression, and increased levels of MeCP2 protein expression in the MPFC and hippocampus of stressed mice (Fig. 2A and B).

Besides, we performed immunofluorescence to stain Nrf2 and MeCP2 in the selected brain regions. Stress significantly decreased the Nrf2 fluorescence intensity, whereas stress significantly increased the MeCP2 fluorescence intensity in the mPFC and dentate gyrus (DG) of the hippocampus. SFN significantly attenuated the reduction of Nrf2 fluorescence intensity and increased MeCP2 fluorescence intensity in the mPFC and DG of the hippocampus in the stressed mice (Fig. 2C-H).

\section{SFN revises Nrf2 and MeCP2 abnormal expression on the microglia of stressed mice}

Base on microglial dominated the CNS injury and repair. Therefore, we try to explore whether SFN could revise Nrf2 and MeCP2 abnormal expression on the microglia of stressed mice. Using the immunofluorescence staining for CD11b (microglia marker) and Nrf2 or MeCP2. The immunofluorescence staining results found that CD11b and Nrf2 or MeCP2 showed a common localization (Fig. 3A and B). Moreover, we found stress significantly increased CD11b and MeCP2 immunoreactivity, whereas stress significantly decreased the Nrf2 immunoreactivity in the MPFC and hippocampus (Fig. 3A and B). In contrast, SFN significantly attenuated the increase of CD11b and the MeCP2 immunoreactivity, whereas SFN significantly attenuated the reduction of Nrf2 immunoreactivity in stressed mice (Fig. 3A and B). The results suggested that SFN may activate BDNF transcription on the microglia.

\section{SFN revises Nrf2 and MeCP2 abnormal expression on the M1-type and M2-type microglia of stressed mice}

In the CNS, the microglial activation is heterogeneous, which can be categorized into two opposite types: M1-type and M2-type. Depended on the phenotypes activation, microglia can produce either cytotoxic or neuroprotective effects [27]. Here, we further explored whether SFN could revise Nrf2 and MeCP2 abnormal expression on the M1-type and M2-type microglia of stressed mice. By using the 
immunofluorescence for iNOS (M1-type microglia marker) and Nrf2 or MeCP2, CD206 (M2-type microglia marker), and Nrf2 or MeCP2. The results found that iNOS and Nrf2 or MeCP2, CD206, and Nrf2 or MeCP2 also showed a common localization (Fig. 4A and B). Stress significantly increased iNOS and MeCP2 immunoreactivity, whereas stress significantly decreased CD206 and Nrf2 immunoreactivity in the mPFC (Fig. 4A and B). Pretreatment of SFN significantly attenuated the increase of iNOS and MeCP2 immunoreactivity, whereas SFN significantly attenuated the decrease of CD206 and Nrf2 immunoreactivity in the mPFC of stressed mice (Fig. 4A and B). Therefore, SFN may activate BDNF transcription on the M2-type of microglia.

\section{Anti-inflammatory effects of SFN in the serum of stressed mice}

Our in vivo studies have found that SFN could correct microglia balance dysfunction in stressed mice. Here, we further explored whether SFN could inhibit pro-inflammatory cytokine release and promote antiinflammatory cytokine release in stressed mice. The serum levels of pro-inflammatory cytokine TNF-a, IL$1 \beta$, IL- 6 , and anti-inflammatory cytokine IL-10 and IL- 4 in mice were measured by using ELSA. We found that serum levels of TNF- $\alpha$, IL-1 $\beta$, and IL- 6 in stressed mice were significantly higher than those of vehicletreated mice (Fig. 5A-C). Pretreatment of SFN significantly attenuated the increased serum levels of TNFa, IL-1 $\beta$, and IL- 6 in stressed mice (Fig. 5A-C). In contrast, the serum levels of IL-10 and IL-4 in stressed mice were significantly lower than those of vehicle-treated mice (Fig. 5D and E). Pretreatment of SFN significantly attenuated the decreased serum levels of IL-10 and IL-4 in stressed mice (Fig. 5D and E). The results suggested that the anti-inflammatory effects of SFN in stressed mice.

\section{The prevention effects of SFN in stressed mice and the dendritic spine density in the MPFC and hippocampus}

The previous research has found that SFN may activate BDNF transcription on the microglia. Here, we try to explore whether SFN activated BDNF transcription on microglia to produce prevention effects and revises abnormal dendritic spine morphology in stressed mice. By employing the CSDS mice model, we found that pretreatment with SFN showed prevention effects in CSDS mice model (Fig. 6A-C). In the social interaction test (no target), the social interaction time has no significant changes in all groups (Fig. 6B). In the social interaction test (target), SFN significantly attenuated the decreased social avoidance time in stressed mice (Fig. 6B). In the sucrose preference test, SFN significantly attenuated the decreased sucrose preference of stressed mice (Fig. 6C). Therefore, SFN showed prevention effects in the stressed mice.

BDNF is a crucial neurotropic factor mediating neural plasticity, and our in vitro and in vivo study found that SFN could promote BDNF transcription by affecting Bdnf transcriptional translation. Here, we hypothesized that the prevention effects of SFN in stressed mice by promoting BDNF transcription to revise abnormal dendritic spine morphology in the MPFC and hippocampus of stressed mice. To confirm this, we employed Thy1-YFP mice, which are spine model mice [28]. After 10 days of social defeat stress, we found that the dendritic spine density significantly decreased in the MPFC and DG of the hippocampus (Fig. 6D and E), pretreatment of SFN significantly attenuated the reduction of the dendritic 
spine density in the mPFC and DG of the hippocampus (Fig. 6D and E). These data suggested the role of SFN regulated BDNF expression contributed to repair synaptic plasticity impairment in the MPFC and hippocampus of stressed mice.

\section{Discussion}

In the current study, the activation of Nrf2 by SFN could promote BDNF transcription resulted in BDNF protein expression on the BV2 cell. SFN revised abnormal BDNF protein expression by attenuating the decreased levels of Nrf2 and increased levels of MeCP2 expression in the stressed mice. Next, we further found that SFN could attenuate the decreased levels of Nrf2 and increased levels of MeCP2 expression on the microglial of stressed mice, and revise microglial dysfunction. Furthermore, SFN attenuated the increased levels of pro-inflammatory cytokine release and decreased levels of anti-inflammatory cytokine release in stressed mice. At last, SFN showed prevention effects in stress mice and revised abnormal dendritic spine morphology in the MPFC and hippocampus. Taken together, the prophylactic effects of SFN in stressed mice by activating BDNF transcription on the microglial and revising abnormal dendritic spine morphology.

The organosulfur compound SFN is considered as the agonist of Nrf2. Recently a study found SFN showed antioxidant and anti-inflammatory effects $[3,4,29]$. The mechanism study revealed that the antioxidant and anti-inflammatory effects of SFN are thought to be activation of the Nrf2 to promote Phase II detoxification enzymes and antioxidant proteins transcription [6, 7]. Recently our study found SFN also showed antipsychotic effects (such as depression and schizophrenia), the mechanism study found that the antipsychotic effects of SFN by activating BDNF/TrkB signaling in the mouse brain $[2,30$, 31]. However, the precise molecular and cellular mechanisms underlying the antidepressant effects of SFN remain unclear. In the current study, using the luciferase and ChIP assays, we confirmed that SFN promoted Nrf2 binds to the Bdnf exon I promoter. Using immunofluorescence for BV2 cells, we observed the SFN-induced redistribution of Nrf2 and MeCP2 for the nucleus. We found that more MeCP2 within the nucleus and the more diffused nuclear staining pattern of Nrf2 in vehicle-treated cells became more punctate upon LPS treatment, and this phenomenon can be reversed by SFN. The western blot assay found that SFN could attenuate the decreased levels of Nrf2, BDNF protein, and increased levels of MeCP2 protein expression in BV2 cells. These data suggest that SFN could induce Bdnf transcription by activating Nrf2 and inhibiting MeCP2 expression on the BV2 cell.

Because BDNF is integral to both the pathophysiology of depression and the therapeutic mechanisms of antidepressants [9-17]. Several studies showed that decreased levels of BDNF, and polymorphisms in the BDNF gene, are associated with major depression. The low plasma BDNF levels have been linked to suicidal behavior in major depression [32]. The reduced BDNF levels were detected in the MPFC and hippocampus of post-mortem brains sample from psychiatric disorder patients [33]. In another study, a decreased mRNA and protein expression of BDNF and TrkB has been found in the hippocampus from suicides' post-mortems samples [34]. In contrast, it is reported that the elevated levels of BDNF have been detected in the parietal cortex of post-mortem patients with major depressive disorder (MDD) who 
received antidepressant treatment compared with MDD-untreated patients [35]. For our in vivo study, SFN showed prevention effects in stressed mice by revising the reduced levels of Nrf2, and increased levels of MeCP2 resulted in BDNF protein expression. These results suggested that SFN has a protected effect by altering BDNF transcriptional translation abnormalities in stressed mice. Besides, for the dendritic spine morphology, we found that SFN attenuated the social defeat stress-induced reduction of dendritic spine density in the MPFC and hippocampus. BDNF protein synthesis is crucial to the structural plasticity of single dendritic spines $[19,36,37]$, suggesting changes in BDNF transcription in the mPFC and hippocampus with altered dendritic spine density in these regions by the administration of SFN is very important. Synaptogenesis is a key function in the mechanism of antidepressants $[19,36,37]$. Therefore, activation of BDNF transcription by the administration of SFN results in alteration of dendritic spine morphology after social defeat stress, are of interest.

Microglia holds states of "classical activation," "alternative activation," and "acquired deactivation," depending on the microenvironment became activation state and received the factor stimulation [27, 38, 39]. The classical activation state of microglia is termed M1-type microglia, which induce iNOS and NFKB pathways and produce various pro-inflammatory cytokines such as TNF-a, IL-1 $\beta$, and IL-6 [27, 40, 41]. M2-type microglia has an alternative activation state and acquired deactivation state, which are induced by IL-4/IL-13 and IL-10/TGF- $\beta$ respectively. M2-type microglia promotes phagocytosis of cell debris and misfolded proteins, results in ECM reconstruction and tissue repair, and supports neuron survival by secreting neurotrophic factors [27, 40, 41]. M2-type microglia regulates by various anti-inflammatory factors and antagonize the M1 pro-inflammatory responses that finally results in immunosuppression and neuron protection [27, 40,41]. In our study, we observed that microglia were activated in the mPFC and hippocampus of stressed mice, the further study found that social defeat stress increased M1-type microglia activation, and decreased M2-type microglia activation in stressed mice. Pretreatment of SFN inhibited pro-inflammatory cytokine release, M1-type microglia activation, and promoted M2-type microglia activation in stressed mice. These results suggested that SFN acted as a protected effect by balancing microglia dysfunction. By using immunofluorescence staining, we found that Nrf2 or MeCP2, and CD11b (microglia maker) shared a common localization. Besides, Nrf2 or MeCP2, and iNOS (M1-type microglia maker) or CD206 (M2-type microglia maker) also shared a common localization, social defeat stress decreased Nrf2 expression and increased MeCP2 expression on the microglia, especially M2-type microglia. Pretreatment of SFN promoted Nrf2 expression and decreased MeCP2 expression on the microglia of stressed mice. Because of our in vitro study found that Nrf2 could activate BDNF transcription resulted in BDNF protein expression on the BV2 cell. Moreover, M2-type microglia coordinated regulation of various anti-inflammatory factors and antagonize the M1 pro-inflammatory responses, resulting in immunosuppression and neuron protection [27, 40,41]. Therefore, our finding suggested that SFN may promote BDNF transcription on the M2-type microglia, showing prevention effect in stressed mice. Perhaps a deeper understanding of the interaction between microglia and BDNF release may facilitate the development of future strategies for depression treatment. However, a detailed study is needed in the further. 


\section{Conclusions}

In conclusion, SFN could promote BDNF transcription by activating Nrf2 and inhibiting MeCP2 expression on the microglia. The prevention effects of SFN by promoting BDNF transcription resulted in BDNF protein expression on the microglia of stressed mice. Alterations in the interaction between microglia and BDNF release may contribute to the protected effects of SFN, and future strategies for depression treatment.

\section{Abbreviations}

BDNF: Brain-derived neurotrophic factor, Nrf2: NF-E2-related factor-2, sulforaphane: SFN, IP: intraperitoneally, CSDS: chronic social defeat stress, TrkB: tropomyosin-receptor-kinase B, mPFC: medial prefrontal cortex, MeCP2: methyl-CpG binding protein-2, YFP: Yellow fluorescent protein, DMEM: Dulbecco's Modified Eagle Medium, LPS: Lipopolysaccharide, ChIP: Chromatin immunoprecipitation, ELISA: enzyme-linked immunosorbent assay, TNF-a: tumor necrosis factor-a, IL-1 $\beta$ : interleukin-1 $\beta$, IL-6: interleukin-6, IL-10: interleukin-10, IL-4: interleukin-4, PVDF: polyvinylidenedifluoride, S.E.M: mean \pm standard error of the mean, DG: dentate gyrus, MDD: major depressive disorder.

\section{Declarations}

\section{Acknowledgements}

The authors are thankful to Dr. Li Zhang at Joint International Research Laboratory of CNS Regeneration, Guangdong-Hong Kong-Macau Institute of CNS Regeneration, Jinan University to provide the Thy1-YFP mice for us. The authors would like to acknowledge the efforts of all the staff who contributed to this study.

\section{Author contributions}

JZ and YS conceived the project, designed the experiments, analyzed the data, and wrote the manuscript. RT and QC performed the social defeat stress model, behavior study, western blot, immunofluorescence and ELSA for the in vivo study. LH and PD performed the luciferase assay, ChIP assay, western blot, and immunofluorescence for the in vitro study. GC, RF, and FX assisted with data analysis and interpretation and critically read the manuscript.

\section{Funding}

This work is supported by the Fundamental Research Funds for the Central Universities (11620425 to JZ), the Science and Technology Jiaxing (Grant No: 2017 AY33030 to PD), the Natural Science Foundation of Guangdong Province (No. 2019A1515010936 to FX).

\section{Availability of data and materials}


The datasets used and/or analyzed during the current study are available from the corresponding author on reasonable request.

\section{Ethics approval and consent to participate}

All experimental procedures were carried out in accordance with the guidelines approved by the Jinan University Institutional Animal Care and Use Committee.

\section{Consent for publication}

Not applicable.

\section{Competing interests}

The authors declare that they have no competing interests.

\section{References}

1. Zhang Y, Talalay P, Cho CG, Posner GH. A major inducer of anticarcinogenic protective enzymes from broccoli: isolation and elucidation of structure. Proc Natl Acad Sci U S A. 1992;89:2399-403.

2. Zhang JC, Yao W, Dong C, Yang C, Ren Q, Ma M, Han M, Wu J, Ushida Y, Suganuma H, Hashimoto K. Prophylactic effects of sulforaphane on depression-like behavior and dendritic changes in mice after inflammation. J Nutr Biochem. 2017;39:134-44.

3. Kobayashi A, Kang MI, Watai Y, Tong KI, Shibata T, Uchida K, Yamamoto M. Oxidative and electrophilic stresses activate Nrf2 through inhibition of ubiquitination activity of Keap1. Mol Cell Biol. 2006;26:221-9.

4. Kobayashi E, Suzuki T, Yamamoto M. Roles nrf2 plays in myeloid cells and related disorders. Oxid Med Cell Longev. 2013;2013:529219.

5. Ma Q. Role of nrf2 in oxidative stress and toxicity. Annu Rev Pharmacol Toxicol. 2013;53:401-26.

6. Suzuki T, Motohashi H, Yamamoto M. Toward clinical application of the Keap1-Nrf2 pathway. Trends Pharmacol Sci. 2013;34:340-6.

7. Suzuki T, Yamamoto M. Molecular basis of the Keap1-Nrf2 system. Free Radic Biol Med. 2015;88:93-100.

8. Yao W, Zhang JC, Ishima T, Dong C, Yang C, Ren Q, Ma M, Han M, Wu J, Suganuma H, et al. Role of Keap1-Nrf2 signaling in depression and dietary intake of glucoraphanin confers stress resilience in mice. Sci Rep. 2016;6:30659.

9. Nestler EJ, Barrot M, DiLeone RJ, Eisch AJ, Gold SJ, Monteggia LM. Neurobiology of depression. Neuron. 2002;34:13-25.

10. Lindholm JS, Castrén E. Mice with altered BDNF signaling as models for mood disorders and antidepressant effects. Front Behav Neurosci. 2014;8:143. 
11. Hashimoto K, Shimizu E, lyo M. Critical role of brain-derived neurotrophic factor in mood disorders. Brain Res Brain Res Rev. 2004;45:104-14.

12. Castrén E. Neurotrophins and psychiatric disorders. Handb Exp Pharmacol. 2014;220:461-79.

13. Bjorkholm C, Monteggia LM. BDNF - a key transducer of antidepressant effects. Neuropharmacology. 2016;102:72-9.

\section{Hashimoto K. Brain-derived neurotrophic factor as a biomarker for mood disorders: An historical} overview and future directions. Psychiatry and Clinical Neuroscience 2010.

15. Hashimoto K. Rapid-acting antidepressant ketamine, its metabolites and other candidates: A historical overview and future perspective. Psychiatry Clin Neurosci. 2019;73:613-27.

16. Hashimoto K. Molecular mechanisms of the rapid-acting and long-lasting antidepressant actions of (R)-ketamine. Biochem Pharmacol. 2020;177:113935.

17. Zhang JC, Yao W, Hashimoto K. Brain-derived Neurotrophic Factor (BDNF)-TrkB Signaling in Inflammation-related Depression and Potential Therapeutic Targets. Curr Neuropharmacol. 2016;14:721-31.

18. Zhang JC, Yao W, Dong C, Yang C, Ren Q, Ma M, Han M, Hashimoto K. Comparison of ketamine, 7,8dihydroxyflavone, and ANA-12 antidepressant effects in the social defeat stress model of depression. Psychopharmacology. 2015;232:4325-35.

19. Zhang JC, Wu J, Fujita Y, Yao W, Ren Q, Yang C, Li SX, Shirayama Y, Hashimoto K. Antidepressant effects of TrkB ligands on depression-like behavior and dendritic changes in mice after inflammation. Int J Neuropsychopharmaco/ 2014, 18.

20. Yang C, Shirayama Y, Zhang JC, Ren Q, Hashimoto K. Regional differences in brain-derived neurotrophic factor levels and dendritic spine density confer resilience to inescapable stress. Int $J$ Neuropsychopharmacol. 2015;18:pyu121.

21. Shirayama Y, Yang C, Zhang JC, Ren Q, Yao W, Hashimoto K. Alterations in brain-derived neurotrophic factor (BDNF) and its precursor proBDNF in the brain regions of a learned helplessness rat model and the antidepressant effects of a TrkB agonist and antagonist. Eur Neuropsychopharmacol. 2015;25:2449-58.

22. Taliaz D, Loya A, Gersner R, Haramati S, Chen A, Zangen A. Resilience to chronic stress is mediated by hippocampal brain-derived neurotrophic factor. J Neurosci. 2011;31:4475-83.

23. Yang B, Yang C, Ren Q, Zhang JC, Chen QX, Shirayama Y, Hashimoto K. Regional differences in the expression of brain-derived neurotrophic factor (BDNF) pro-peptide, proBDNF and preproBDNF in the brain confer stress resilience. Eur Arch Psychiatry Clin Neurosci. 2016;266:765-9.

24. Zhang JC, Yao W, Dong C, Han M, Shirayama Y, Hashimoto K. Keap1-Nrf2 signaling pathway confers resilience versus susceptibility to inescapable electric stress. Eur Arch Psychiatry Clin Neurosci. 2018;268:865-70.

25. Martinowich K, Hattori D, Wu H, Fouse S, He F, Hu Y, Fan G, Sun YE. DNA methylation-related chromatin remodeling in activity-dependent BDNF gene regulation. Science. 2003;302:890-3. 
26. Li C, Zhao B, Lin C, Gong Z, An X. TREM2 inhibits inflammatory responses in mouse microglia by suppressing the PI3K/NF-kappaB signaling. Cell Biol Int. 2019;43:360-72.

27. Tang Y, Le W. Differential Roles of M1 and M2 Microglia in Neurodegenerative Diseases. Mol Neurobiol. 2016;53:1181-94.

28. Popescu IR, Le KQ, Palenzuela R, Voglewede R, Mostany R. Marked bias towards spontaneous synaptic inhibition distinguishes non-adapting from adapting layer 5 pyramidal neurons in the barrel cortex. Sci Rep. 2017;7:14959.

29. Kometsi L, Govender K, Mofo Mato EP, Hurchund R, Owira PMO. By reducing oxidative stress, naringenin mitigates hyperglycaemia-induced upregulation of hepatic nuclear factor erythroid 2related factor 2 protein. J Pharm Pharmacol 2020.

30. Shirai Y, Fujita Y, Hashimoto K. Effects of the antioxidant sulforaphane on hyperlocomotion and prepulse inhibition deficits in mice after phencyclidine administration. Clin Psychopharmacol Neurosci. 2012;10:94-8.

31. Shirai Y, Fujita Y, Hashimoto R, Ohi K, Yamamori H, Yasuda Y, Ishima T, Suganuma H, Ushida Y, Takeda M, Hashimoto K. Dietary Intake of Sulforaphane-Rich Broccoli Sprout Extracts during Juvenile and Adolescence Can Prevent Phencyclidine-Induced Cognitive Deficits at Adulthood. Plos One 2015, 10.

32. Kim JM, Stewart R, Kim SW, Yang SJ, Shin IS, Kim YH, Yoon JS. Interactions between life stressors and susceptibility genes (5-HTTLPR and BDNF) on depression in Korean elders. Biol Psychiat. 2007;62:423-8.

33. Karege F, Vaudan G, Schwald M, Perroud N, La Harpe R. Neurotrophin levels in postmortem brains of suicide victims and the effects of antemortem diagnosis and psychotropic drugs. Brain Res Mol Brain Res. 2005;136:29-37.

34. Banerjee R, Ghosh AK, Ghosh B, Bhattacharyya S, Mondal AC. Decreased mRNA and Protein Expression of BDNF, NGF, and their Receptors in the Hippocampus from Suicide: An Analysis in Human Postmortem Brain. Clin Med Insights Pathol. 2013;6:1-11.

35. Sheldrick A, Camara S, llieva M, Riederer P, Michel TM. Brain-derived neurotrophic factor (BDNF) and neurotrophin 3 (NT3) levels in post-mortem brain tissue from patients with depression compared to healthy individuals - a proof of concept study. Eur Psychiatry. 2017;46:65-71.

36. Magarinos AM, Li CJ, Gal Toth J, Bath KG, Jing D, Lee FS, McEwen BS. Effect of brain-derived neurotrophic factor haploinsufficiency on stress-induced remodeling of hippocampal neurons. Hippocampus. 2011;21:253-64.

37. Govindarajan A, Rao BSS, Nair D, Trinh M, Mawjee N, Tonegawa S, Chattarji S. Transgenic brainderived neurotrophic factor expression causes both anxiogenic and antidepressant effects. Proc Natl Acad Sci USA. 2006;103:13208-13.

38. Miron VE, Boyd A, Zhao JW, Yuen TJ, Ruckh JM, Shadrach JL, van Wijngaarden P, Wagers AJ, Williams A, Franklin RJM, Ffrench-Constant C. M2 microglia and macrophages drive oligodendrocyte differentiation during CNS remyelination. Nat Neurosci. 2013;16:1211-8. 
39. Kigerl KA, Gensel JC, Ankeny DP, Alexander JK, Donnelly DJ, Popovich PG. Identification of Two Distinct Macrophage Subsets with Divergent Effects Causing either Neurotoxicity or Regeneration in the Injured Mouse Spinal Cord. J Neurosci. 2009;29:13435-44.

40. Block ML, Zecca L, Hong JS. Microglia-mediated neurotoxicity: uncovering the molecular mechanisms. Nat Rev Neurosci. 2007;8:57-69.

41. Zhou XL, Spittau B, Krieglstein K. TGF beta signalling plays an important role in IL4-induced alternative activation of microglia. Journal of Neuroinflammation 2012, 9.

\section{Figures}
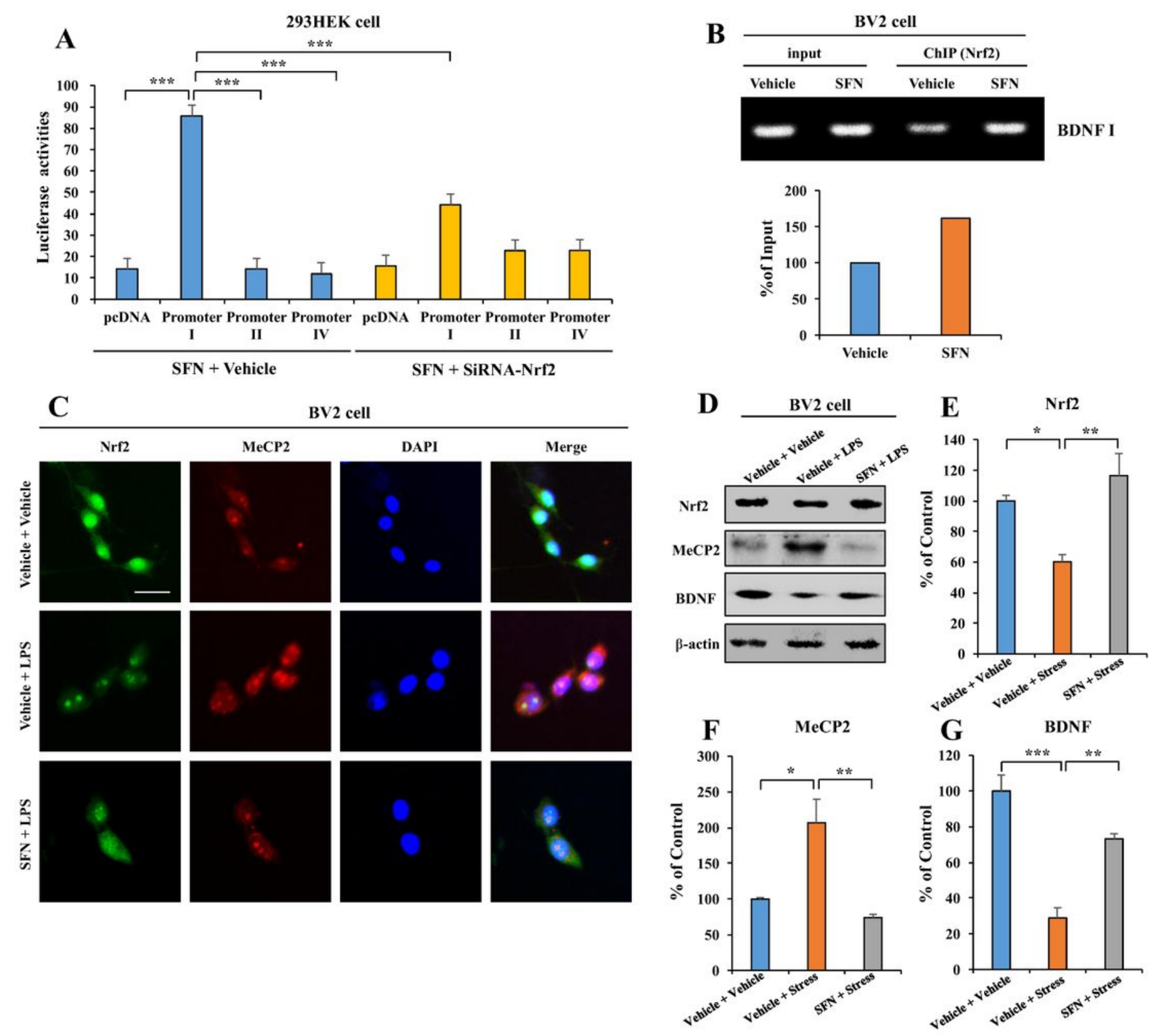

Figure 1 
Activation of Nrf2 by SFN resulted in BDNF transcription on the BV2 cell A: The luciferase assay for BDNF I, II, and IV promoter. The HEK293 cell was treated with SFN or Siran-Nrf2 for 24 hours. The luciferase assay results of pcDNA, BDNF II, and IV promoter were compared with BDNF I promotor (Mean $\pm \mathrm{SEM}, \mathrm{n}=$ 3 per group, one-way ANOVA, $\left.{ }^{\star *} p<0.001\right)$. B: ChIP-PCR assays for BDNF I promoter. The Nrf2 proteinDNA crosslinking samples were obtained from the BV2 cells treated with SFN or vehicle via coimmunoprecipitating with anti-Nrf2 or IgG antibodies. PCR was carried out by using BDNF exon I promoter primer. C: The immunofluorescence for Nrf2 and MeCP2. The SFN or LPS was treated for BV2 cells for 24 hours. The immunofluorescence was performed for Nrf2 and MeCP2. Scar bar $50 \mu \mathrm{m}$. D: The representative image for the Western blot of Nrf2, MeCP2, and BDNF. E: The quantitative result for Nrf2. F: The quantitative result for MeCP2. G: The quantitative result for BDNF. (Mean $\pm S E M, n=3$ per group, oneway ANOVA, ${ }^{*}<0.05, * \star p<0.01$ and ${ }^{\star * *} p<0.01$ ). 

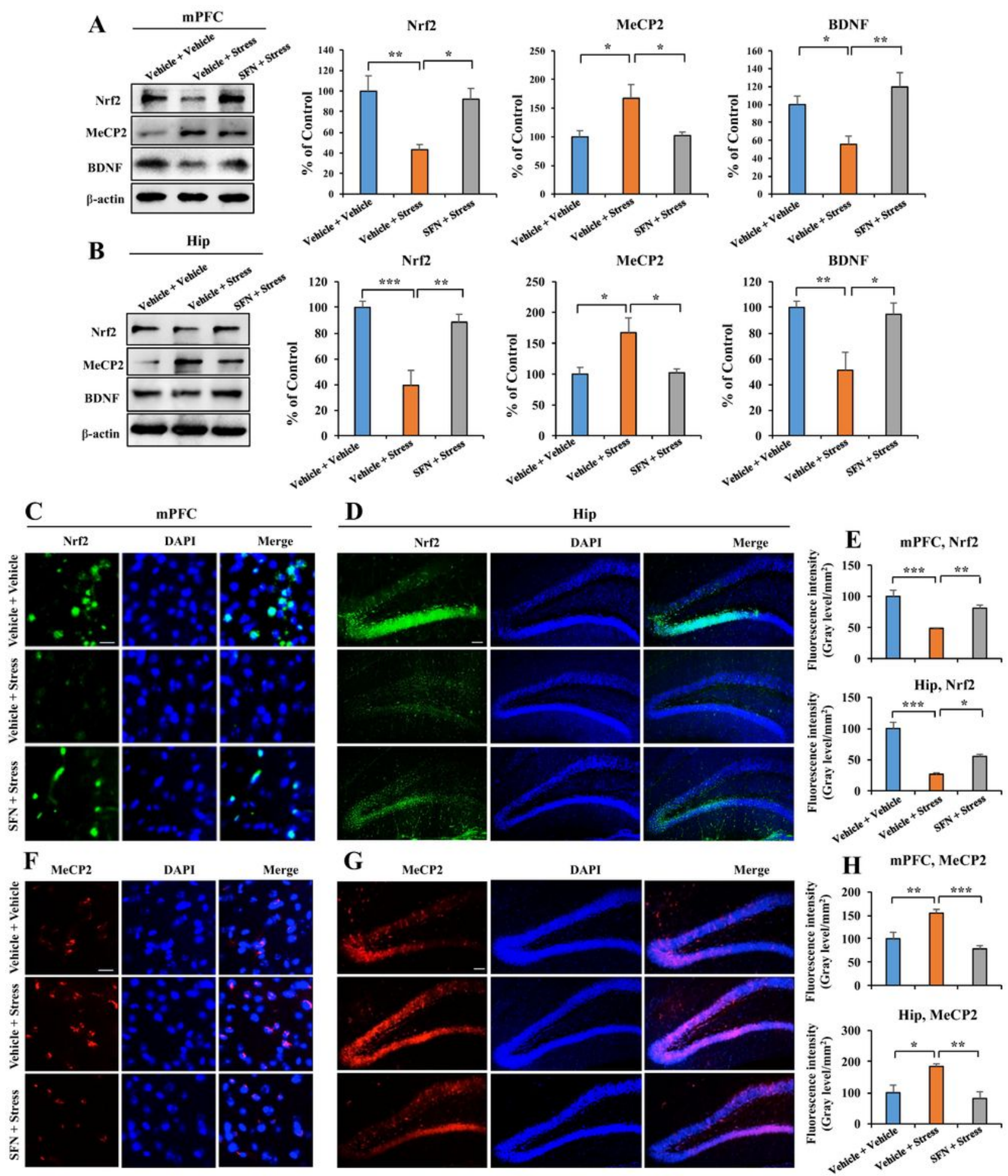

\section{Figure 2}

SFN attenuates the decreased levels of Nrf2 and increased levels of MeCP2 expression, revising abnormal BDNF expression in the stressed mice $A$ and $B$ : The western blot analysis for Nrf2, MeCP2, and BDNF in mPFC and hippocampus of stress mice (Mean \pm SEM, $n=4$ per group, one-way ANOVA, ${ }^{*}<$ $\left.0.05,{ }^{*} \mathrm{p}<0.01\right)$. C - E: The immunofluorescence staining for Nrf2 in mPFC and hippocampus of stressed mice. F - H: The immunofluorescence staining for MeCP2 in mPFC and hippocampus. $\mathrm{E}$ and $\mathrm{H}$ : The 
quantification analysis of Nrf2 and MeCP2 fluorescence intensity in mPFC and hippocampus of stressed mice. Values represent the mean \pm standard error of the mean (SEM; $n=4$ per group, one-way ANOVA, * $p$ $<0.05, * * p<0.01$, and $* * * p<0.001)$.
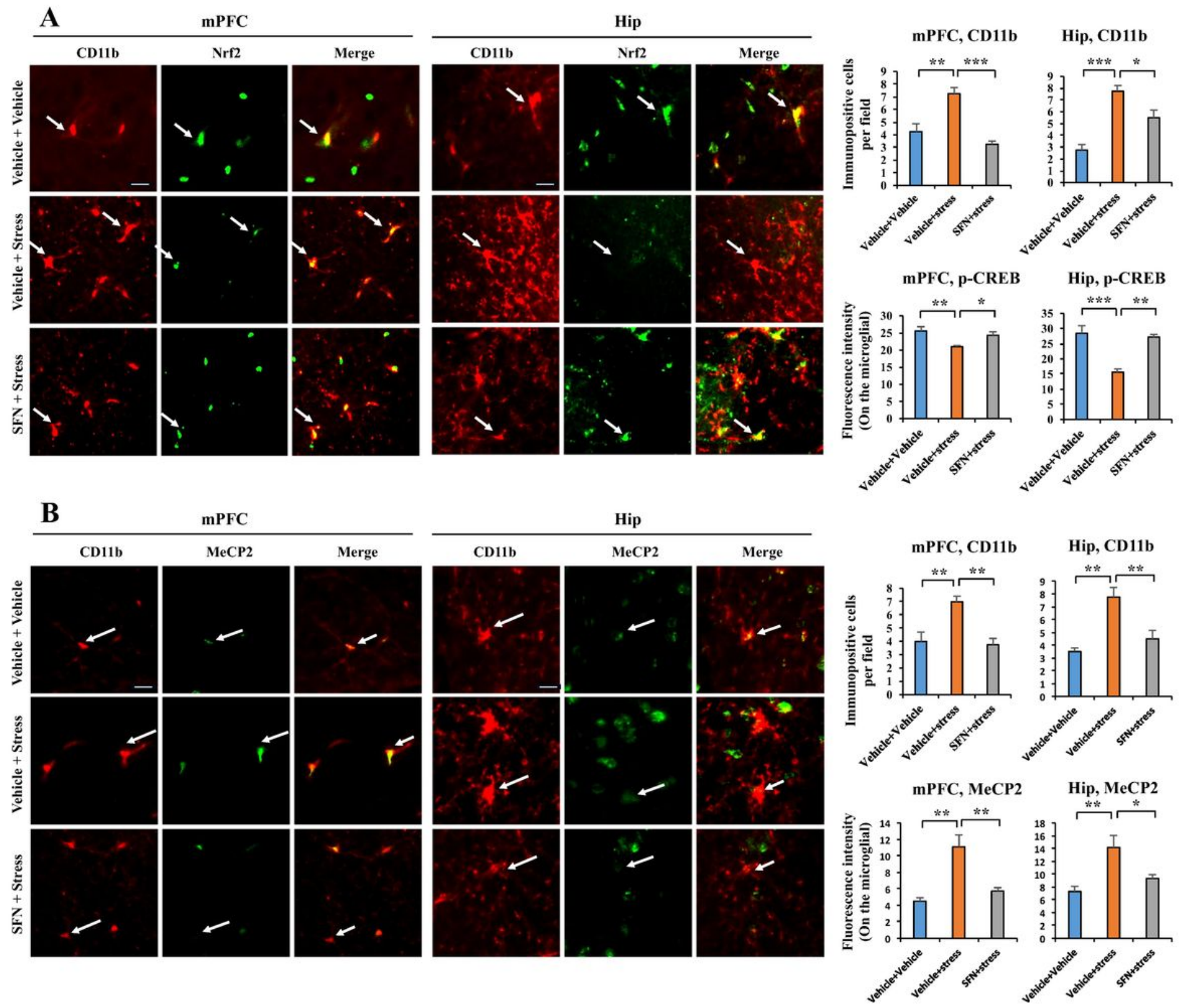

Figure 3

SFN revises Nrf2 and MeCP2 abnormal expression on the microglia of stressed mice A: The representative photomicrographs for the microglial marker (CD11b) and Nrf2 in the mPFC and hippocampus, scar bar $50 \mu \mathrm{m}$. The quantification analysis of activation microglial and Nrf2 fluorescence intensity. Values represent the mean \pm standard error of the mean (SEM; $n=4$ per group, one-way ANOVA, ${ }^{*} p<0.05,{ }^{*} p<0.01$, and $\left.{ }^{* * *} p<0.001\right)$. B: The representative photomicrographs for the microglial marker (CD11b) and MeCP2 in the hippocampus, scar bar $50 \mu \mathrm{m}$. The quantification analysis of activation microglial and MeCP2 fluorescence intensity. Values represent the mean \pm standard error of the mean 
(SEM; $n=4$ per group, one-way ANOVA, ${ }^{*} p<0.05,{ }^{*} p<0.01$, and $\left.{ }^{* *} \mathrm{p}<0.001\right)$. The white arrows indicated the nuclear Nrf2 or MeCP2 staining preserved in some microglial.
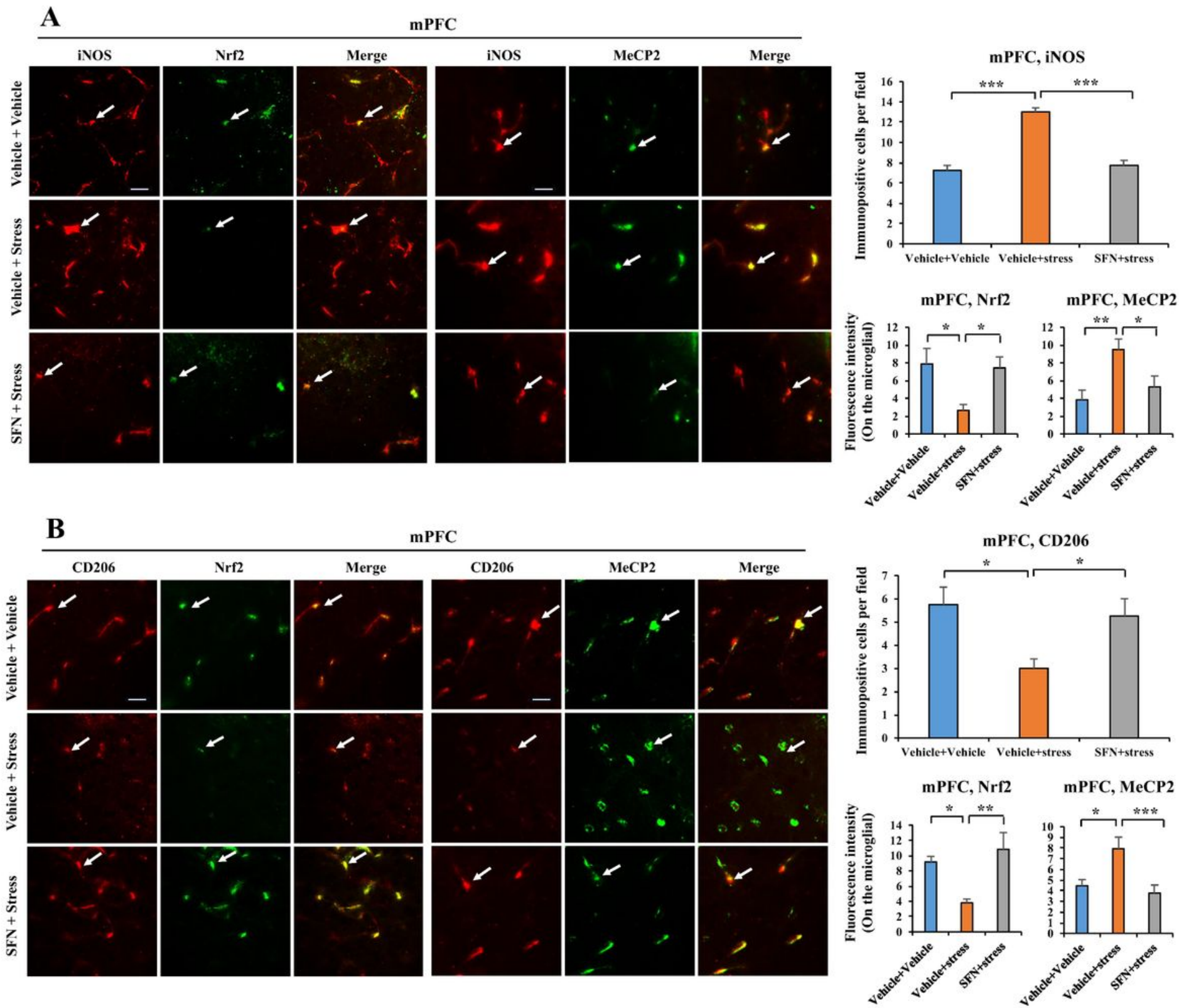

\section{Figure 4}

SFN revises Nrf2 and MeCP2 abnormal expression on the M1/M2-type microglia of stressed mice A: The representative photomicrographs for M1-type microglial marker (iNOS), Nrf2, and MeCP2 in the mPFC, scar bar $50 \mu \mathrm{m}$. The quantification analysis of Nrf2 and MeCP2 fluorescence intensity. Values represent the mean \pm standard error of the mean (SEM; $n=4$ per group, one-way ANOVA, ${ }^{*}<<0.05,{ }^{*} p<0.01$, and $\star \star \star p<0.001)$. The white arrows indicated the nuclear Nrf2 or MeCP2 staining preserved in some M1-type microglial. B: The representative photomicrographs for M2-type microglial marker (CD206), Nrf2, and MeCP2 in the mPFC, scar bar $50 \mu \mathrm{m}$. The quantification analysis of Nrf2 and MeCP2 fluorescence intensity. Values represent the mean \pm standard error of the mean (SEM; $n=4$ per group, one-way ANOVA, 
${ }^{*} \mathrm{p}<0.05,{ }^{*} \mathrm{p}<0.01$, and $\left.{ }^{* \star *} \mathrm{p}<0.001\right)$. The white arrows indicated the nuclear Nrf2 or MeCP2 staining preserved in some M2-type microglial.
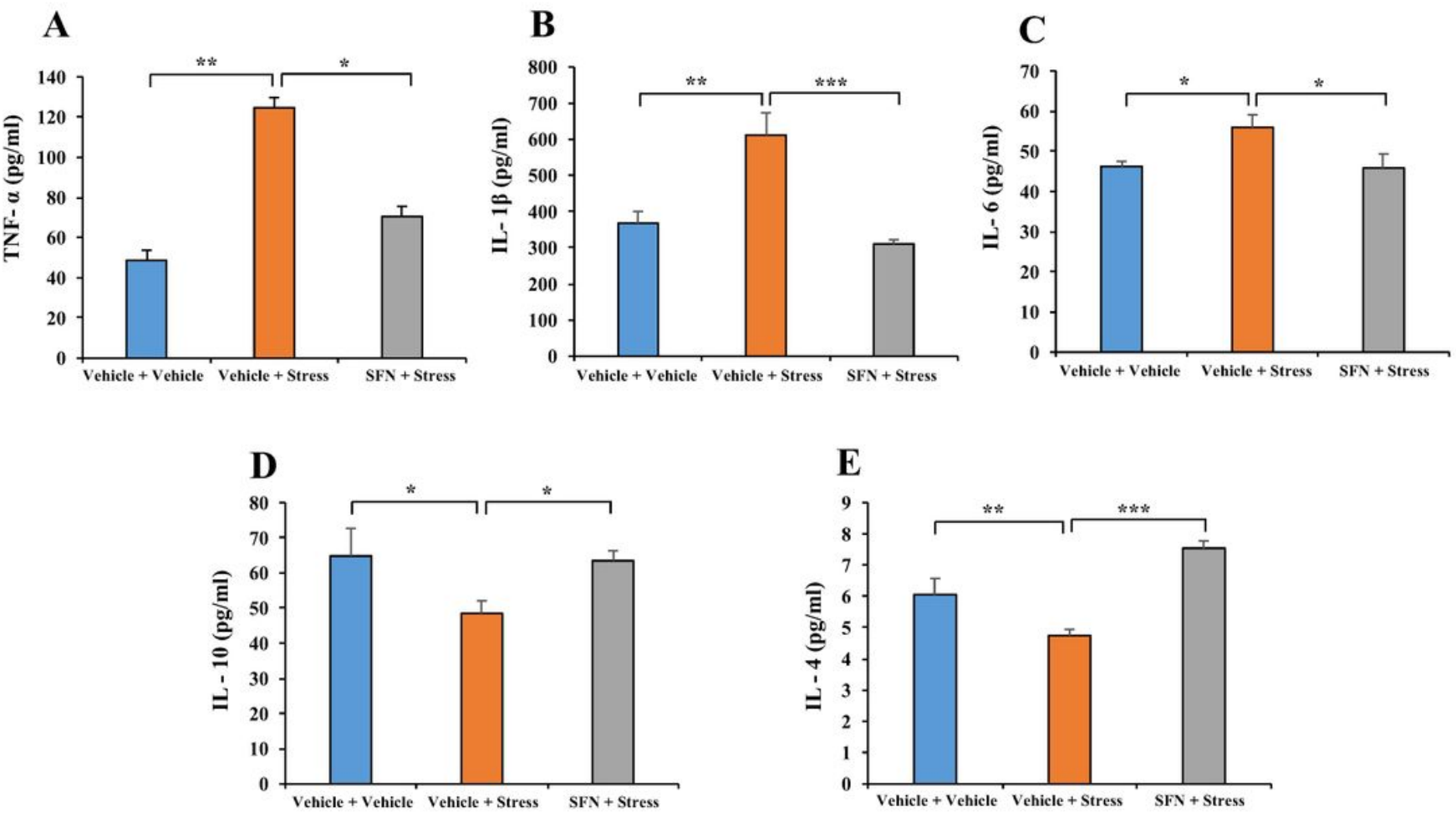

\section{Figure 5}

Anti-inflammatory effects of SFN in the serum of stressed mice A: Serum levels of TNF-a on day 13. Values represent the mean \pm standard error of the mean (SEM; $n=7$ per group, one-way ANOVA, ${ }^{*} p<0.05$, $\left.{ }^{*} p<0.01\right)$. B: Serum levels of IL-1 $\beta$ on day 13. Values represent the mean \pm standard error of the mean (SEM; $n=7$ per group, one-way ANOVA, ${ }^{\star} p<0.05,{ }^{* \star} p<0.01$ ). C: Serum levels of T IL-6 on day 13 . Values represent the mean \pm standard error of the mean (SEM; $n=7$ per group, one-way ANOVA, ${ }^{\star} p<0.05$, ** $p<$ 0.01). D: Serum levels of T IL-10 on day 13. Values represent the mean \pm standard error of the mean (SEM; $n=6$ per group, one-way ANOVA, ${ }^{\star} p<0.05,{ }^{*} p<0.01$ ). E: Serum levels of T IL-4 on day 13. Values represent the mean \pm standard error of the mean (SEM; $n=6$ per group, one-way ANOVA, ${ }^{\star} p<0.05$, ** $p<$ 0.01). 

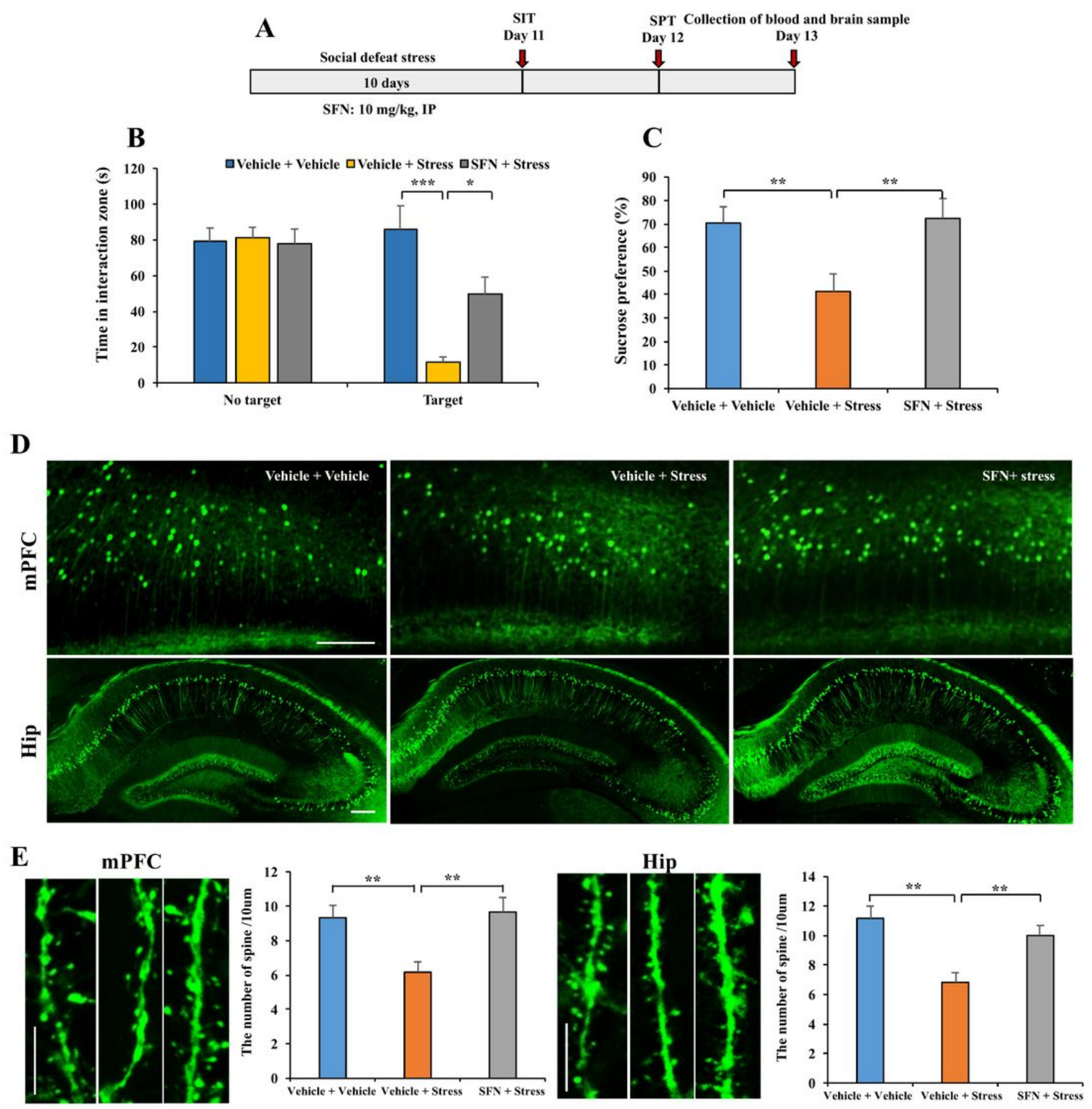

\section{Figure 6}

The prevention effects of SFN in stressed mice and the dendritic spine density in the MPFC and hippocampus A: The schedule of behavior tests and treatment. B: The social interaction test for no target and target time (Mean \pm SEM, $n=8$ per group, one-way ANOVA, ${ }^{*}<0.05, * \star p<0.01$ ). C: The sucrose preference test (Mean $\pm S E M, n=8$ per group, one-way ANOVA, $* \star p<0.01)$. D: The representative photomicrographs for low magnification for the mPFC and hippocampus, scar bar $50 \mu \mathrm{m}$. E: The 
representative photomicrographs for high magnification for the mPFC and hippocampus, scar bar $10 \mu \mathrm{m}$. Values represent the mean \pm standard error of the mean $\left(n=4\right.$ per group, one-way ANOVA, $\left.{ }^{\star \star} p<0.01\right)$. 